\section{«THE IRON BRIDGE»: EL PRIMER PUENTE METALICO}

\section{Documentación gráfica proporcionada por IAN DOBBIE}

Adaptación y traducción del texto* por J. SALAS

$565-42$

\section{SINOPSIS}

Se describe en este articulo, mediante reportaje gráfico y la traducción parcial de un folleto editado por el Museo "The Iron Bridge», el contexto histórico $y$ las circunstancias técnicas en las que se proyectó y ejecutó tan famoso puente.

La inauguración del puente tuvo lugar el 1 de enero de 1781. El arquitecto autor del proyecto fue Thomas Farnolls Pritchard, realizando los elementos fundidos Abraham Darby III.

\section{ANTECEDENTES}

El puente fundido Coalbrookdale, primero de su género en el mundo, es el más importante de los monumentos industriales de la Gran Bretaña. Ha sido siempre un espectáculo, una curiosidad, una prueba palpable de la pericia y osadia de los maestros fundidores del dieciocho en la región de Shropshire.

Cuando se proyectó "The Iron Bridge", la garganta del río Severn ya era, desde hacía dos centurias, una zona industrial activa. La explotación, a gran escala, del carbón para su envio a lo largo del río habia sido la base de su prosperidad durante los siglos XVI y XVII. Hacia 1750 comienza la rápida expansión del mercado del hierro de East Shropshire, que para finales del XVIII hace de este distrito la mayor área de producción en fundición, de Gran Bretaña. La marcha de la actividad industrial era tal que un buen número de barcos para grandes cargas hacian su trayecto a través del río. Caliza desde Benthall Edge y mineral de hierro procedente de Ladywood Ilegaban a los hor-

\footnotetext{
- Traducción parcial tomada del folleto: «The Iron Bridge. A short history of the first iron bridge in the world». Publicado por "The Ironbridge Gorge Museum Trust 1979", texto original de Barrie Trinder. Museum Guide n. ${ }^{\circ} 3.01$.
}

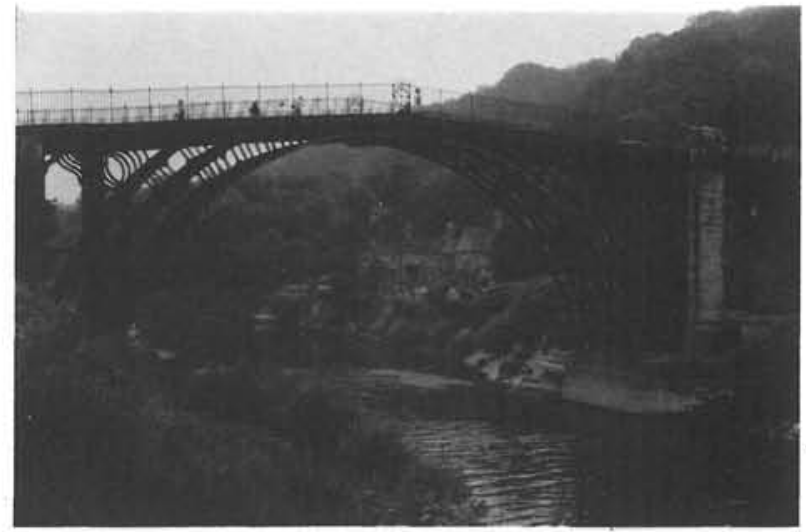

nos de Coalbrookdale. Muchas personas cruzaban el rio para ir al trabajo, asistir a ceremonias religiosas o diversiones. El puente más cercano estaba entre Builwas y Bridgworth, y la falta de un puente en Gorge suponia un fuerte problema, tanto para las actividades industriales como para las de tipo social. No sorprende, pues, que desde 1750 , al menos, se hable entre los vecinos de Coalbrookdale de construir un puente.

El tráfico de barcazas en el Severn era tan intenso que hacia necesario un puente de un solo tramo. Por otra parte, las escarpadas orillas de la garganta imposibilitaban la construcción de una estructura de varios vanos de crecimiento gradual, a la manera de lo construido en piedra por John Gwynne en Atcham entre 1769-71 o por Thomas Telford a base de madera en Cressage en 17991801. Salvar el río Severn a la altura de Coalbrookdale era, por consiguiente, algo que sobrepasaba los limites de la tecnología de la época. Construir un puente metálico no era una cuestión de mania, capricho o alarde, sino la aplicación de una nueva tecnologia para la solución de un dificil problema concreto.

\section{ORIGENES DEL PROYECTO}

En 1773 Thomas Farnolls Pritchard, arquitecto de Shrewsbury, escribió al maestro fundidor John Wilkinson, sugiriéndole la construcción de un puente metálico en Severn Gorge. Pritchard habia nacido en 1723 y era hijo de un carpintero de Shrewsbury. En 1770 era responsable de un buen número de importantes edificios en Shropshire y condados limitrofes, y tenía muchas conexiones con los principales maestros fundidores.

En 1756 diseñó el monumento a la primera mujer de John Wilkinson en la parroquia de Wrexham y posteriormente trabajó en la casa de Wilkinson en Broseley. Se ocupó de trabajos de restauración para el tercero de los Abraham Darby en Hay 

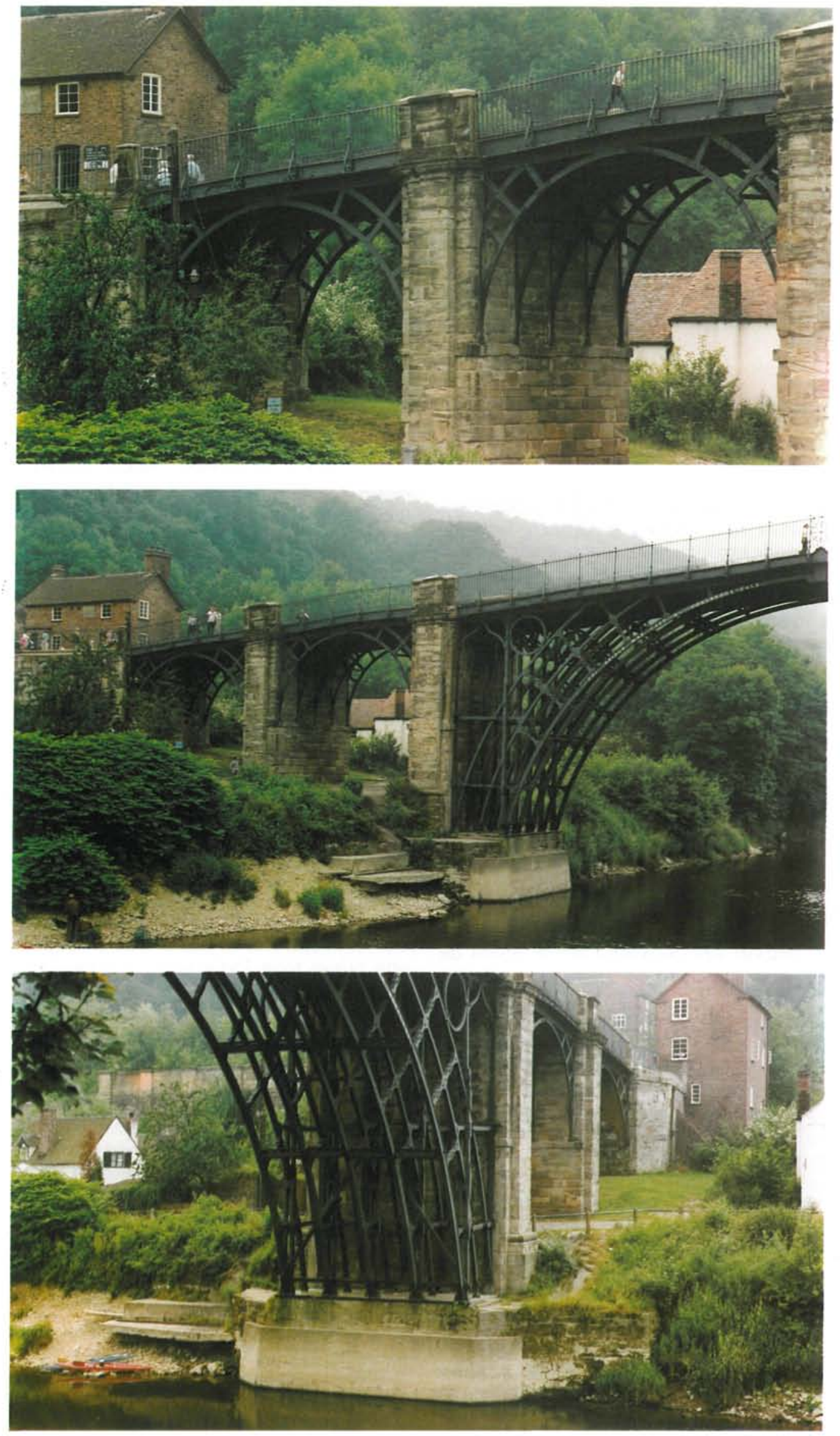
Farm (Madeley), y encargó chimeneas diseñadas por él para ser fundidas por la Compañia Coalbrookdale de Benthall Hall en Corvedale. Tenia también una considerable experiencia en el diseño de puentes. Presentó los planos (no los finalmente empleados), para el nuevo "English Bridge" en Shrewsbury en 1760 y fue supervisor de la obra de mamposteria del puente sobre el río Teme en Bringewood Forge, cerca de Ludlow. Durante 1773-75 diseñó el puente de tres vanos sobre el Severn en Stourport.

John Wilkinson era, en la década de 1770, uno de los maestros fundidores más prominentes de Inglaterra, con trabajos en Denbighhire y Sttaffordshire, asi como en Willey, Shropshire. Era un innovador, por lo que su participación en un proyecto como el que describimos concuerda totalmente con su carácter. Parece que esto fue lo que le hizo dar los primeros pasos para hacer realidad la propuesta de Pritchard. En febrero de 1774 el periódico "West Midlands" recogia que las poblaciones de Broseley y Madeley se proponian solicitar del Parlamento la construcción de un puente metálico de un arco sobre el río Severn cerca de Coalbrookdale. En septiembre del mismo año un grupo de personas interesadas en el proyecto se reunió en la taberna de Broseley. El emplazamiento del puente estaba definido: el arranque sur estaria en la parroquia de Benthall; el norte, junto a la casa de Thomas Crumpton en Madeley Wood. Abraham Darby III de Coalbrookdale fue nombrado tesorero del proyecto. Se tomaron las medidas para asegurar la consecución del decreto del Parlamento. En la lista de firmantes junto a John Wilkinson y Abraham Darby III figuran muchos de los responsables de las industrias locales, entre ellos Edward Blakeway, socio de Wilkinson en Willey y uno de los fundadores de la fábrica de Ioza Coalport; Edward Harries, Lord del Condado de Benthall; John Thursfield, de Benthall Hall, un cirujano y propietario de una mina y John y Charles Guest, miembros de la antigua mina de la familia Broseley y fundadores de los talleres Dowlais en el sur de Gales. Thomas Farnolls Pritchard, también firmante, fue comisionado en la reunión para preparar un diseño del puente. Sus primeros planos, preparados en octubre de 1775 , eran para un puente de cuatro arcadas o costillas con una luz de $120 \mathrm{ft}(36,60 \mathrm{~m})$. Abraham Darby III aceptó construir el puente y presupuestó el coste total de la estructura, incluido el suministro de la fundición en 3.200 libras.

El decreto parlamentario para la construcción del puente se obtuvo en febrero de 1776, pero durante los dieciocho meses siguientes los firmantes tuvieron dudas sobre el proyecto. En mayo de 1776 se rescindió el contrato a Darby para la construcción del puente, anunciándose públicamente el concurso de proyectos para un puente de un vano de piedra, ladrillo o madera. No se presentó nin-
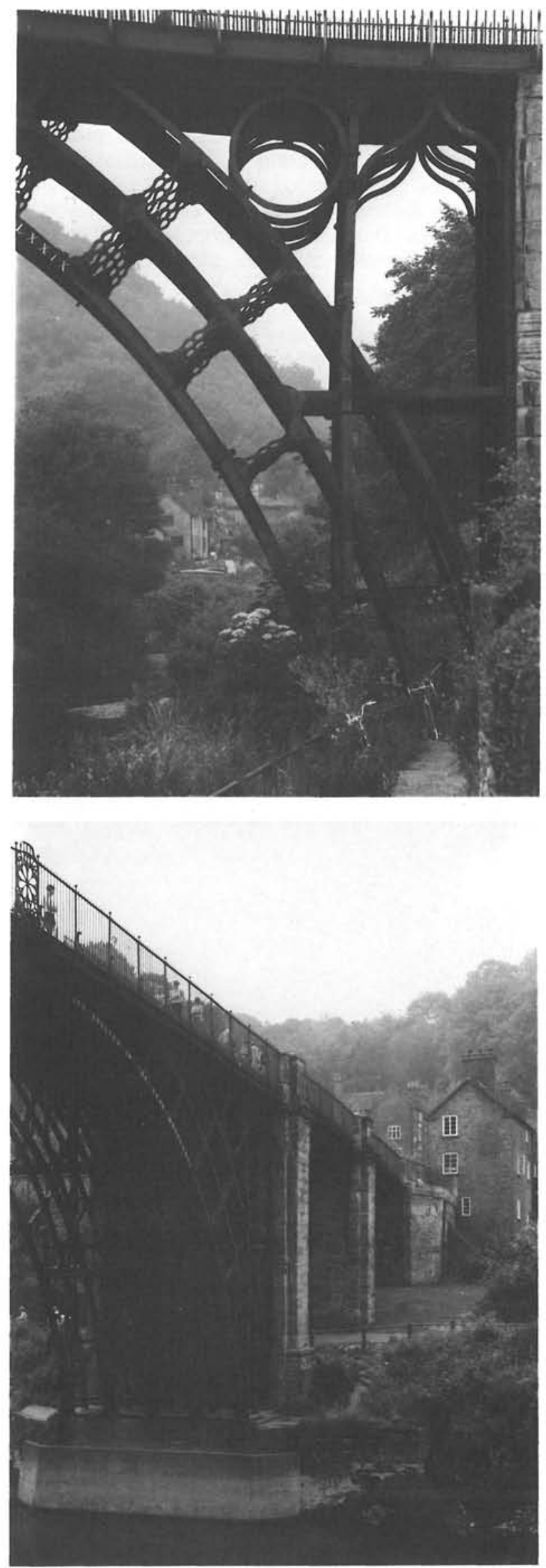
gún concursante y en julio de 1776 se autorizó a Pritchard para preparar la maqueta de un puente metálico. Se originó un cisma entre los firmantes: Abraham Darby III y sus seguidores Pritchard, Wilkinson y un molinero llamado Leonard Jennings, habian determinado construir un puente metálico y disponian de la mayoría de las acciones. No obstante, el resto de los accionistas, mayor en número, se inclinaban por construir una estructura más ortodoxa. Durante el invierno de 1776 se produjo una nueva retención, no resuelta hasta octubre de 1777.

El diseño del puente había sido alterado desde los tiempos del primer proyecto, en el que se fijaba una luz de $120 \mathrm{ft}(36,60 \mathrm{~m})$. En julio de $1777 \mathrm{la}$ luz se fijó en $90 \mathrm{ft}(27,45 \mathrm{~m})$. Posiblemente, la luz se aumentó a $100 \mathrm{ft}$. 6 inchs $(30,65 \mathrm{~m})$ para acomodarse al trazado de los senderos del municipio. El plano de julio de 1777 coincide esencialmente con el puente existente en la actualidad.

\section{LA CONSTRUCCION}

Finalizado el diseño, la construcción del puente corrió a cargo de Abraham Darby III. El se encargó del pago de los trabajos, asi como de suministrar los materiales y equipos de construcción. Las cuentas del proyecto quedaban bajo su responsabilidad personal.

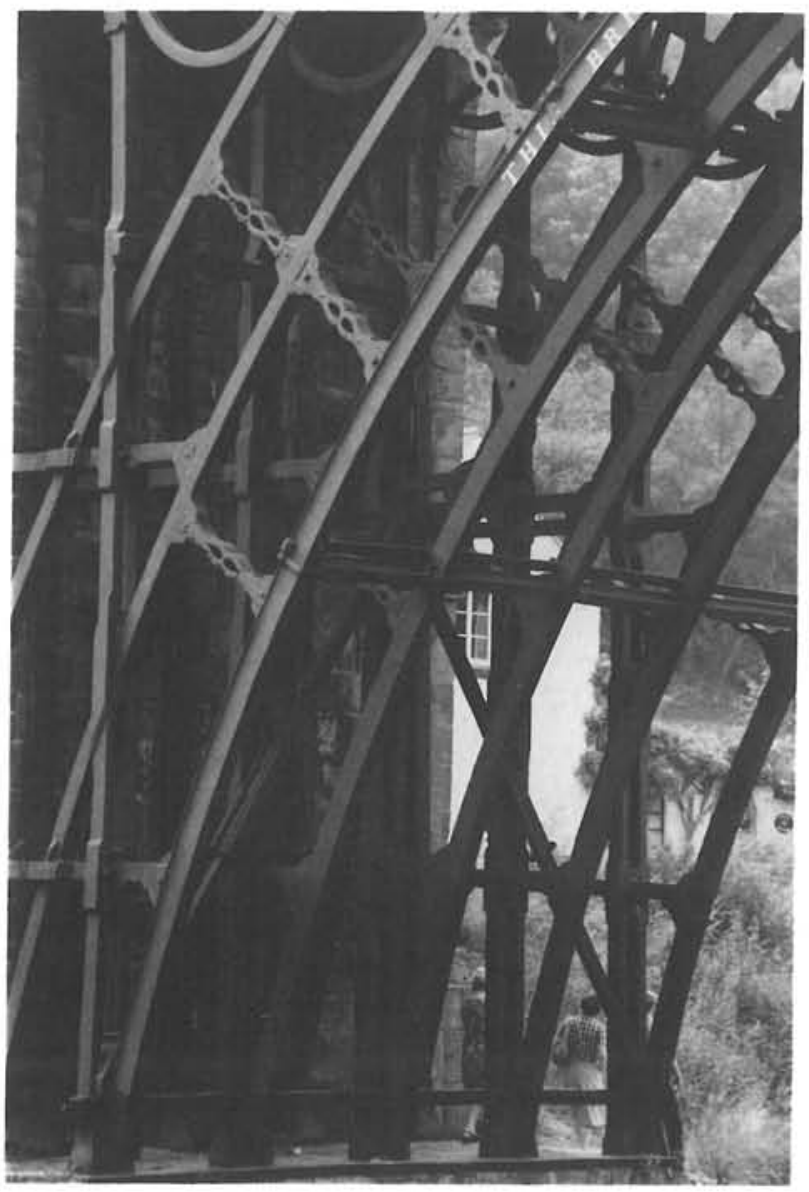

Los trabajos de construcción se iniciaron en noviembre de 1777 aunque fueron muy pocos los empleados que trabajaron ese invierno. Durante el verano y otoño de 1778 fueron probablemente entre 20 y 30 las personas empleadas, en doble jornada durante algunos periodos. La tarea fundamental era la construcción de los estribos; su finalización se celebró con una fiesta a finales de octubre. El ritmo de actividad bajó durante el invierno pero, para la primavera de 1779 , estaban finalizados los trabajos de preparación para recibir la estructura. Observaciones realizadas por John Wesley, tras una visita a la zona el 26 de marzo, señalan que las arcadas estaban entonces en espera para su montaje. En abril, un mínimo de 40 hombres trabajaba en la obra, probablemente levantando el andamiaje. Durante junio y los dias 1 y 2 de julio se montaron dos de las arcadas principales. El acabado de la estructura general fue entonces más rápido. El gasto de nueve guineas en cerveza, que figura con fecha 23 de octubre, seguramente corresponde a la celebración del final de montaje, y en noviembre se retiró el andamiaje. Durante el periodo de las obras el tráfico de barcazas por el Severn continuó normalmente.

La finalización del puente se prolongó más de lo esperado. Hubo dificultades en la ejecución de la calzada y en la conexión con los caminos que causaron bastantes problemas. Durante 1780, trabajaron unos 20 hombres hasta que el puente se

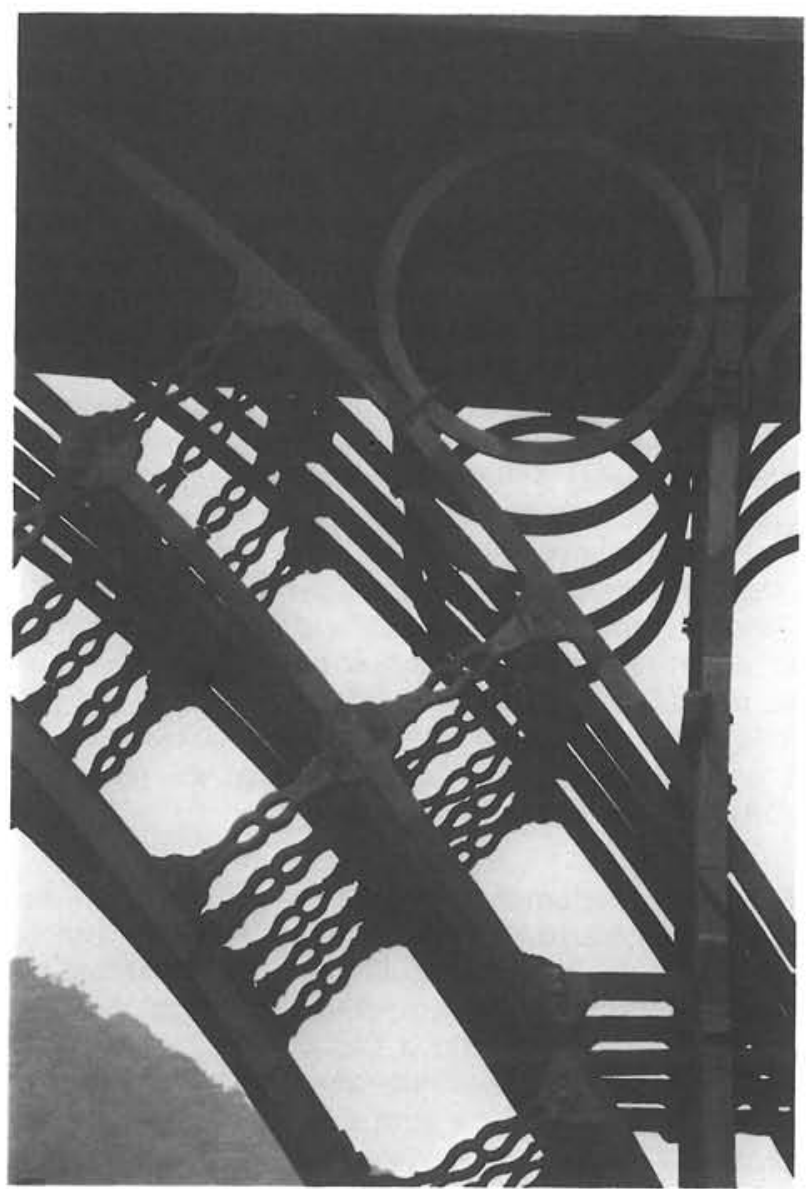




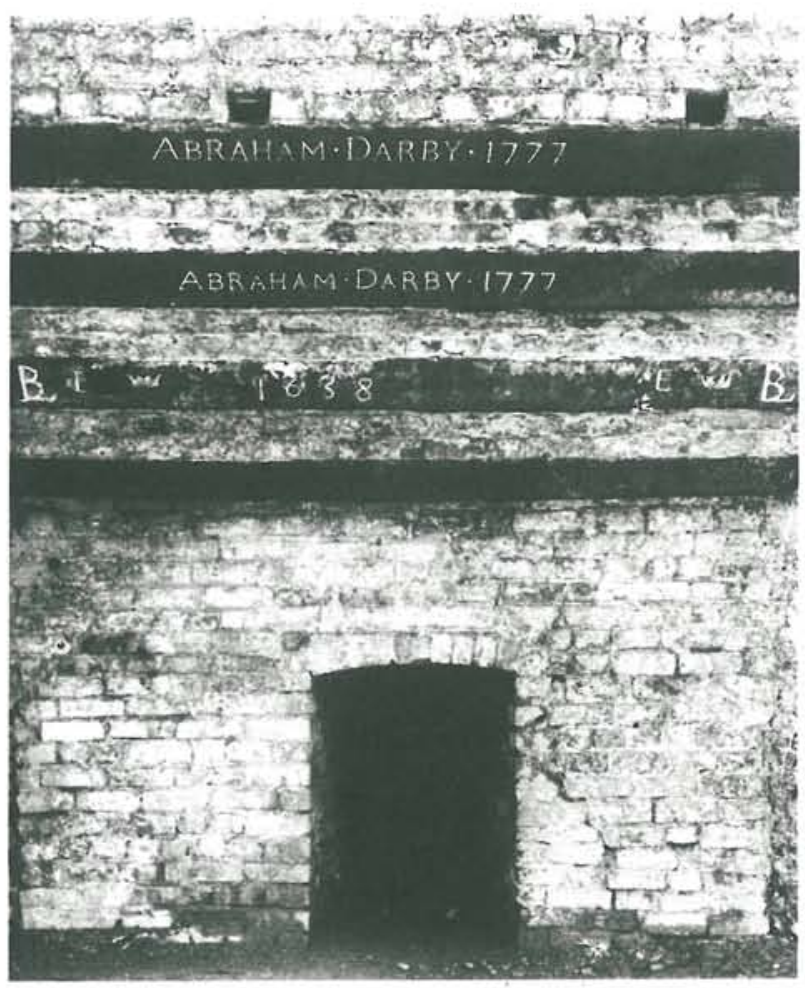

inauguró el día primero del año de 1781. El coste total de la construcción, excluyendo los materiales con los que se realizó, ascendió a 2737 libras, 4 chelines, 10 peniques, lo que contrasta con las escasas 550 libras estimadas como presupuesto en octubre de 1775. El coste de los elementos fundidos se desconoce hasta el presente.

\section{¿DONDE SE REALIZARON LOS ELEMENTOS DE FUNDICION?}

No existe duda alguna en la actualidad sobre el lugar en el que se fundieron los elementos metálicos del puente. Según la tradición, los nervios estructurales se realizaron en el alto horno de la fundición Coalbrookdale, aproximadamente a una milla y un cuarto del emplazamiento. Cada uno de los principales elementos pesa $5,15 \mathrm{t}$; resultaba pues un verdadero reto mover estos elementos con los medios de transporte propios de 1779. Esta complicación hizo pensar a muchas personas que los elementos no se habian fundido en Coalbrookdale, sino en un horno al aire libre emplazado junto a la implantación del puente o bien en los hornos cercanos de Madeley Wood, que habian sido adquiridos por Abraham Darby III en 1776. Los hechos de carácter histórico tienden a confirmar la tradición de que la fundición de la estructura se realizó en Coalbrookdale. Justamente después de la decisión final de ejecutar un puente en hierro fundido, un miembro de la familia Darby escribió: "supongo que se fundirá en el "dale" por el tío Abraham que tendrá la dirección de todo".

Diferentes contemporáneos de los hechos narraron que el puente, sin ningún tipo de dudas, se fundió en el valle de Coalbrook.
Mayor relevancia histórica tiene la evidencia arqueológica existente en el horno de Coalbrookdale: las vigas indicadoras de la importante reconstrucción realizada en 1777 , que indican que se realizaban algunos proyectos importantes. Para estos años no tenía sentido, desde el punto de vista económico, el ampliar los hornos de Coalbrookdale debido a que los costes de transporte eran superiores a los de otros talleres propiedad de sus socios en Horsehay y Ketley, a no ser que hubiese una razón especial para hacerlo. La cantidad de hierro empleada en el puente era equivalente en la época a la capacidad de producción durante un periodo de tres o cuatro meses; por ello, era esencial una adaptación de las instalaciones para mantener la cadencia de suministro.

\section{CARACTERISTICAS DEL PUENTE METALICO}

El puente construido es bastante menos innovador en el empleo del hierro que el propuesto originalmente por Pritchard en 1775. Es, de alguna manera, consecuencia de la práctica del momento a base de otros materiales. La forma y proporciones generales del puente se acercan mucho a un buen número de puentes contemporáneos de un vano, realizados a base de mamposteria y se asemeja particularmente al puente de piedra realizado por Thomas Farnolls Pritchard en 1772, que salva el rio Teme en Bringewood Forge. Los detalles constructivos y la forma de unión de las partes recuerdan mucho las prácticas usuales en carpinteria. Las placas de base se unen entre si mediante juntas en cola de milano. Las claves de los cinco arcos se aseguran mediante cuñas. Los radiales se sujetan a los arcos principales mediante juntas de carga. El peso del hierro del puente es de $378 \mathrm{t}$, bastante más superior que cualquier puente similar de las mismas dimensiones. La falta de dominio de la nueva tecnología por parte de los constructores les hizo ser cautelosos, prefiriendo la seguridad a la economía de materiales.

Existe una constante tendencia de ciertos estratos del terreno en la garganta del Severn a deslizar hacia el río. No pasó mucho tiempo antes de que movimientos de este tipo empezasen a dañar el puente. En diciembre de 1784 se apreciaron grietas sobre el arco en la parte del lado de Benthall. En 1791 se reparó la parte dañada y en años pos-

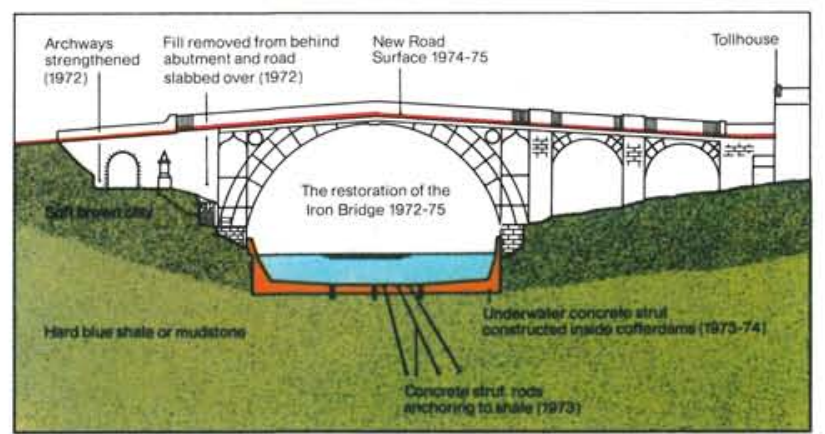


teriores se repararon los estribos de la parte sur. Entre 1802-04 el terraplenado macizo de paramento de piedra fue sustituido por dos pequeños arcos de madera, los cuales a su vez se reemplazaron por los arcos metálicos actuales en 1821. Las partes metálicas se protegieron en 1788 con un barniz suministrado por Lord Dundonald de la Cía. British Tar, el mismo que se utilizó en los cercanos puentes metálicos de Calcutts y Benthall.

\section{EI siglo $X X$}

El puente metálico Coalbrookdale es bastante más antiguo que todos sus inmediatos sucesores. Este y el puente de Cound, que data de 1797, son seguramente los únicos puentes metálicos del siglo XVIII que permanecen intactos. Sin embargo, no ha sido capaz de hacer frente al incremento de la densidad del tráfico durante los últimos setenta años. En 1909 la presión en este sentido se mitigó con la apertura del puente de hormigón, "Free Bridgen, aproximadamente a media milla aguas abajo. El tráfico que pasaba sobre el puente metálico hasta entonces tenia que pagar tasas, por lo que muchos optaban por el desvio. En 1931 el puente se cerró al tráfico de vehículos, permaneciendo abierto para los peatones que tenian que pagar un penique en la casa de tasas situadas en la parte sur.

En 1950 se ocupó de su administración el Shropshire County Council, quedando abolido el pago de impuestos. Hacia tiempo que era necesario acometer reparaciones sustanciales, que planteaban bastantes problemas de ingeniería. Estas se acometieron en 1971-2, sufragando los gastos de las mismas el Ironbridge George Museum Troust.

El trabajo de reconstrucción se inició en 1972, erigiendo una estructura de hormigón y acero en el estribo norte, el cual habia sido relleno previamente con cenizas y escorias. Entre 1973 y 1974 se construyó, en el lecho del rio, un arco invertido de hormigón al objeto de ayudar a mantener los estribos. En años posteriores se reemplazó el firme y la casa de recaudación se transformó en centro de información con una exposición de ilustraciones de la historia del puente, en la que se puede ver el retrato de Thomas Farnolls Pritchard, asi como uno de los moldes originales de madera utilizados para fundir piezas de los arcos y una relación de los primeros cuadros del puente.

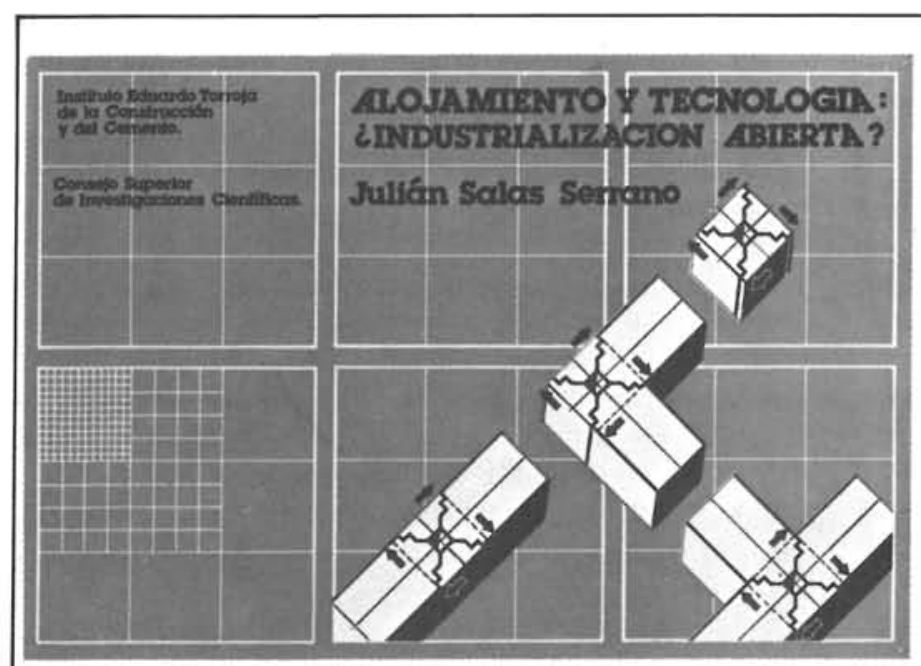

\section{ALOJAMIENTO Y TECNOLOGIA: ¿INDUSTRIALIZACION ABIERTA?}

JÚLIAN SALAS, ING. IND. (I.E.T.c.c.)

Un volumen de 160 páginas, 109 figuras y 16 tablas. Tamaño $240 \times 168 \mathrm{~mm}$. Encuadernado en rústica. Precios: España, 1.200 ptas; extranjero, 17 \$ USA.

\section{SUMARIO:}

Prólogo Prof. G. Ciribini.

\section{Introducción}

Capítulo 1.- La industrialización en las proclamas y manifiestos de arquitectura.

Capítulo 2. - ¿Réquiem por la construcción industrializada?

Capítulo 3.-Algunos conceptos básicos.

Capítulo 4.- ¿Proyecto tradicional, construcción industrializada?

Capitulo 5.-Componentes.

Capítulo 6. - La coordinación dimensional hoy.

Capítulo 7.-Flexibilidad, intercambiabilidad y catálogos. Capítulo 8. - Industrialización, normativa y calidad.

Capítulo 9.-Reflexiones finales.

publicación del

INSTITUTO EDUARDO TORROJA 


\section{Resúmenes}
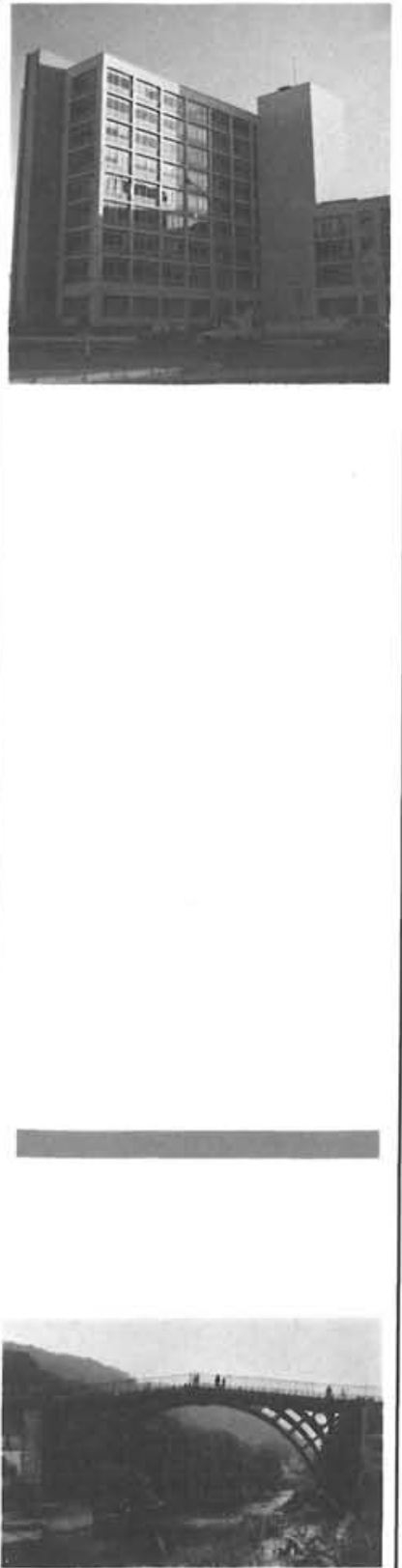

Edificio de

Servicios

Múltiples

Eduardo Méndez,

Arquitecto función de las necesidades de espacio de los distintos puestos de trabajo, se ha realizado este edificio que engloba las numerosas dependencias de varios Ministerios e Institutos. compone de dos niveles de servicios; una planta baja destinados a oficinas, enlazados por el núcleo de comunicaciones verticales. del falso techo, base de 1,25 x 1,25 metros.

\section{"The Iron Bridge": el primer puente} metálico proporcionada por IAN DOBBIE

Adaptación y traducción del texto

por J. Salas las que se proyecto realizando los elementos fundidos Abraham Darby III.

\section{Resumes \\ Bâtiment polyvalent Valladolid (Espagne) \\ Eduardo Méndez, \\ Summaries \\ Multiple Services \\ Building \\ Valladolid/Spain}

Valladolid/Espafía

A partir de los módulos de la Junta Coordinadora de edificios administrativos, establecidos en

En esencia, la construcción se sótano para aparcamiento y destinada a cafeteria y salón de actos; dos cuerpos elevados, Para la estructura, asi como para el dimensionamiento de los puestos de trabajo y de los huecos de ventana, e incluso, del despiece del pavimento y

se ha partido de un módulo

Documentación gráfica

Se describe en este articulo, mediante reportaje gráfico y la traducción parcial de un folleto editado por el Museo aThe Iron Bridgen, el contexto histórico y las circunstancias técnicas en

La inauguración del puente tuvo lugar el 1 de enero de 1781. El arquitecto autor del proyecto fue Thomas Farnolls Pritchard, architecte

A partir des modules du Conseil de Coordination des bâtiments administratifs, établis en fonction des besoins d'espace des différents postes de travail, a été réalisé ce bâtiment qui englobe les nombreuses dépendances de plusieurs Ministères et Instituts.

Par essence, le bâtiment se compose de deux niveaux de sous-sol destinés au parking et les services, d'un

rez-de-chausseée destiné à la

cafeteria ot la salle de réunions, de deux corps élevés destinés aux bureaux, le tout uni par le noyau de communications verticales.

On est parti diun module base de $1,25 \times 1,25 \mathrm{~m}$ pour l'exécution de la structure, ainsi que pour le dimensionnement des postes de travail et des baies, et même, de la division du revêtement de sol et du faux plafond.

\section{"The Iron Bridge»: le premier pont métallique}

Documentation graphique fournie par IAN DOBBIE Adaptation et traduction du texte par J. Salas

On décrit dans cet article, moyennant un reportage graphique et la traduction partielle d'une brochure publiée par le Musée "The Iron Bridge", le contexte historique et les circonstances techniques dans lesquels a été conçu et construit ce fameux pont.

L'inauguration du pont a eu lieu le 1 er janvier 1781 . L'architecte auteur du projet a été Thomas Farnolls Pitchard et les éléments fondus ont étè réalisés par Abraham Darby III. the first metalic
Eduardo Méndez,

Architect

From the modules of the Coordinating Committee of administrative buildings,

established in accord with

requirements of space of the different jobs, this building has been constructed to globalize the numerous dependencies of various Ministries and Institutes.

In essence, the construction is composed of two cellar levels for parking and services; a

lower floor for the cafeteria and meeting hall; two upper floors for offices, connected with the nucleous of vertical communications.

For the structure, as likewise for the dimensioning of the jobs and the windows and even the paving of the false ceiling. the basic module was $1.25 \mathrm{x}$ 1.25 metres.

"The Iron Bridgew: bridge

Graphic documentation provided by

IAN DOBBIE

Adaptation and translation of the text by J. Salas

In this article there is described, through a graphic report and the partial translation of a leaflet edited by the Museum "The Iron Bridge", the historical context and the circumstances in which there was projected and executed such a famous bridge.

The bridge was inaugurated on 1st January 1781 . The architect of the Project was Thomas Farnolls Pritchard, the basic elements being carried out by Abraham Darby III.

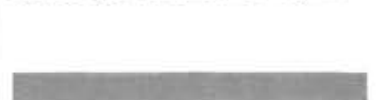

